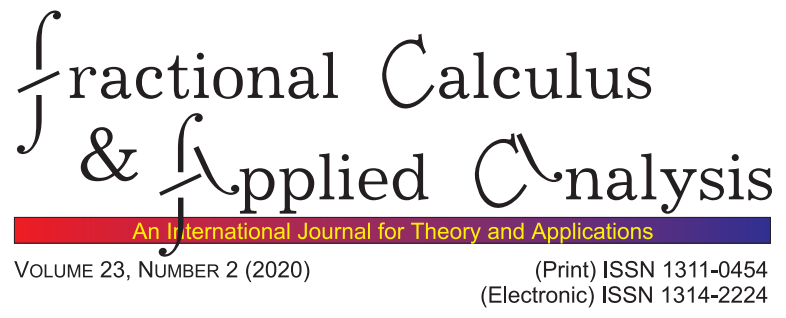

\title{
TUTORIAL SURVEY
}

\section{POROUS FUNCTIONS - II}

\author{
Iryna Trymorush, Igor Podlubny
}

\begin{abstract}
A new mathematical tool, porous functions, has been suggested recently. In this paper operations with porous functions are further discussed alongside with computational tools, which are necessary for evaluation of porous functions, their visualization, and basic operations with such functions in two- and three-dimensional cases.
\end{abstract}

MSC 2010: Primary 26A33; Secondary 76S99, 65C99

Key Words and Phrases: porous functions, porous intervals, composite materials, complex structures and systems, MATLAB

\section{Introduction}

Recently a new approach to modeling the structure of real materials have been introduced [1, based on a new kind of functions - porous functions, with possible applications to modeling various types of real-world materials (granular, porous, composite, etc.) and structures (city agglomerations, forests, etc.) and dynamical processes in such materials and structures. This approach was presented at several conferences [2, 3, 4] in order to verify its viability and potential applications.

In this paper, we further and in more details explain the notions related to porous functions and basic operations with them, including visualizations, computations, and example of code for using the Porous Functions Toolbox (PFT) for MATLAB [2, 5, 6].

(C) 2020 Diogenes Co., Sofia pp. 307-323, DOI: 10.1515/fca-2020-0015

DE GRUYTER 


\section{Cells, Grains, and Pores}

As it was introduced in [1, a porous interval is divided into subintervals. A subinterval, consisting of a bounding box (a minimal rectangular container (in three-dimensional case, it is a parallelepiped) which tightly encloses a grain/pore, is denoted as a cell. The same term is used for the abstract class cell used in PFT for representing grains and pores in twoand three-dimensional cases.

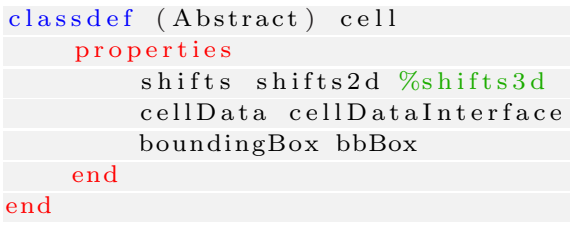

The shifts2d (shifts3d for 3d case) property contains a relative position of a cell inside a porous interval. The width $(\delta x[1])$ and shape of a grain/pore is defined by the cellData property of a class type inherited from cellDataInterface. This allows the creation of any type of cell: of selected standard shapes (circle/sphere, rectangle/parallelepiped, square/cube) or custom shape (irregular, or user-defined shape).

Since cellDataInterface requires only coordinate points of a grain/pore, all details of generating are hidden into derived classes. Thus, PFT contains two separate classes: grain (for creating filled cells - an analogy of some physical material of self-similar structure, such as sand) and pore (for creating void cell to represent foam-like materials). For instance, an ellipse-shaped grain (Fig. 2.1) or pore (Fig. 2.2) can be formed by using ellipseCellData (ellipsoidCellData for 3d case).

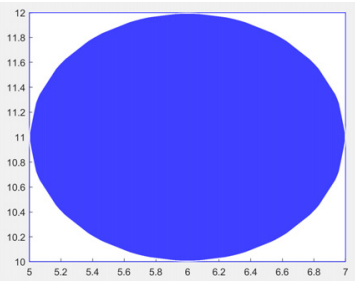

(a) $2 \mathrm{D}$ case

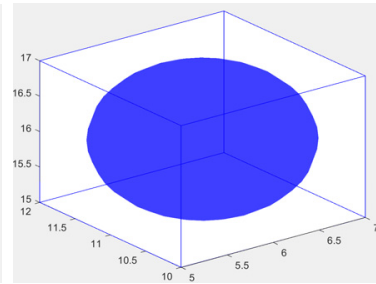

(b) $3 \mathrm{D}$ case

FIG. 2.1: Grains and their cells 


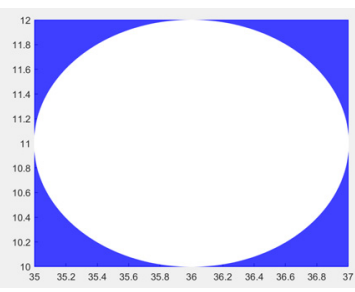

(a) $2 \mathrm{D}$ case

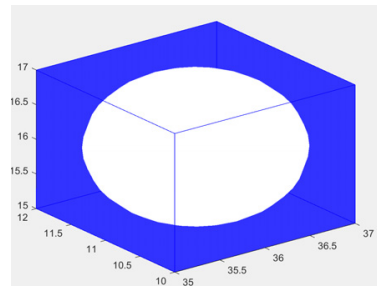

(b) $3 \mathrm{D}$ case

Fig. 2.2: Pores and their cells

Let us illustrate how to create cells shown on Fig. 2.1 and Fig. 2.2 using PFT.

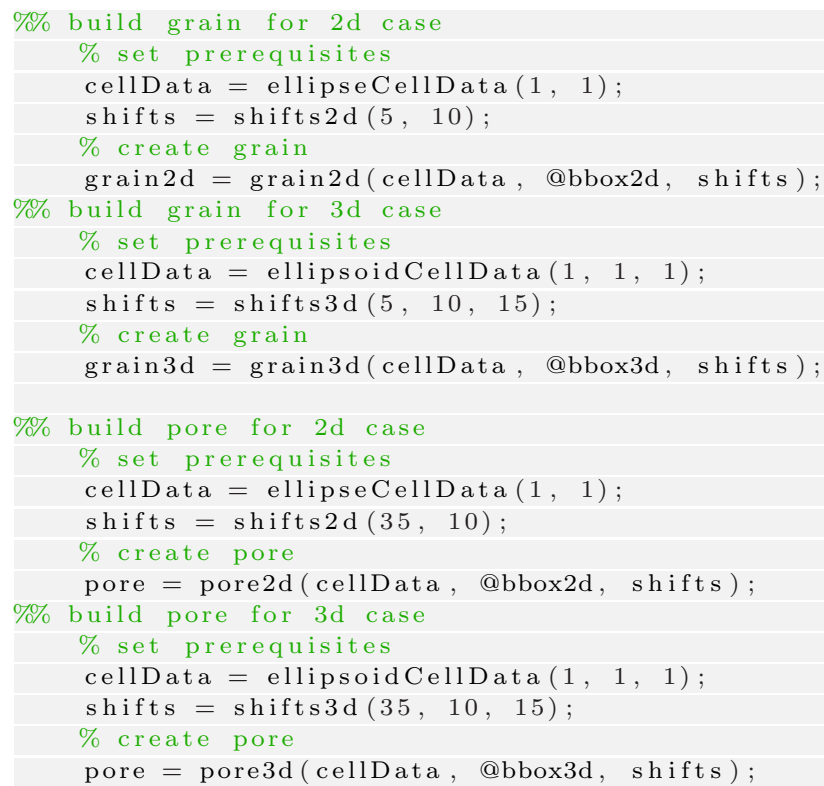

Grains/pores are essential components of porous intervals, as they provide the characteristics used in describing the properties and behavior of the structure as a whole.

\section{Porous Intervals}

3.1. Notation. A porous interval is denoted as [1]

$$
I_{p}=\{[a, b], \delta x, \mu(x)\},
$$

where $a$ and $b$ are the lower and the upper bounds (we can think of them as lines in the two-dimensional case, or planes in the three-dimensional case), 
$\delta x$ is the grain/pore width, and $\mu(x)$ is the rule describing the distribution of grains/pores (either stochastic or deterministic).

Let also denote a grain/pore height as $\delta y$ and consider $\mu(x)$ as $\mu(x ; h, \delta y)$, where $h=|b-a|$ is the height of $I_{p}$. This $\mu(x ; h, \delta y)$ generates a set of $K$ integer numbers, which we denoted $J$ (indices of cells in $I_{p}$ ), where

$$
J=\{k: k \in E(\mu(x ; h, \delta y))\},
$$

where $E$ is the range of the function $\mu(x ; h, \delta y)$, representing the distribution rule based on $h$ and $K, K=\lfloor h / \delta y\rfloor$ is the number of cells that fit in $I_{p}$. Thus, a porous interval can be described as an indexed family of cells with an indexed set $J$

$$
I_{p}=\left\{C_{j}\right\}_{j \in J},
$$

where $C_{j}$ is a set of cells, containing only one cell (3.4).

$$
\left|C_{j}\right|=1 \text {. }
$$

3.2. Creating porous intervals in PFT. An algorithm for creating and handling a porous interval object $I_{p}$ in PFT is based on the cells described in Section 2, and custom classes and interfaces for $I_{p}$ are provided in the PFT. The class pi2d (and pi3d for the three-dimensional case) is the basic one, which allows the creation of $I_{p}$ and manipulations with it: visualization (draw), refill (regenerate indices of cells), performing of basic operations. It is characterized by the following properties:

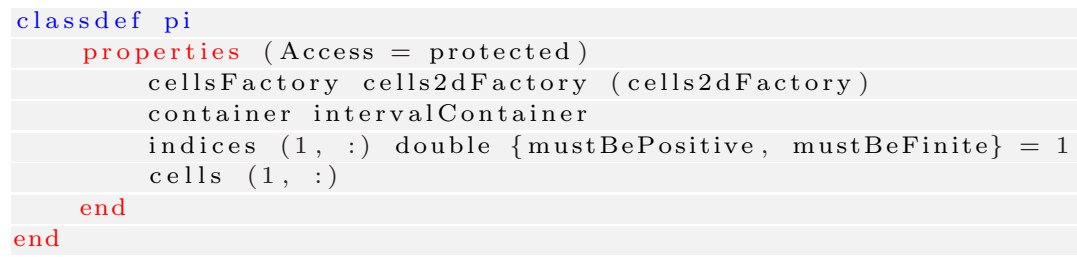

The cellsFactory property is a factory that generates a cell of defined shape and size. It also contains methods for calculating the size characteristics of a porous interval.

The container property represents the constraints of $I_{p}$ based on the cell sizes ( $\delta x, \delta y$ and eventually $\delta z$ in the three-dimensional case) and the interval height $h$. It has a rectangular shape (or parallelepiped in the threedimensional case), but can be customized as necessary.

The indices property represents an array with the indices of the cells positions, distributed following the distribution rule $\mu(x ; h, \delta y)$. This class also provides necessary auxiliary internal properties and methods, which we do not need to list here.

The basic algorithm for creating a porous interval $I_{p}$ is the following:

- define the cell type (shape, size), 
- define the interval $[a, b]$ to build a container for the porous interval,

- define a distribution rule/law $\mu(x ; h, \delta y)$, and generate unique indices of cells of the porous interval,

- place the cells to corresponding calculated positions.

This algorithm is implemented in the method fillinterval of $p i$ class and as a result initiates the cells property.

How it works can be illustrated by running the following code snippet, which generates $I_{p_{2} d}$ and $I_{p_{3} d}$ (Fig. 3.1), filled by the grains described in Section 2 (i.e. elliptical grain with dimensions equal to 1 ):

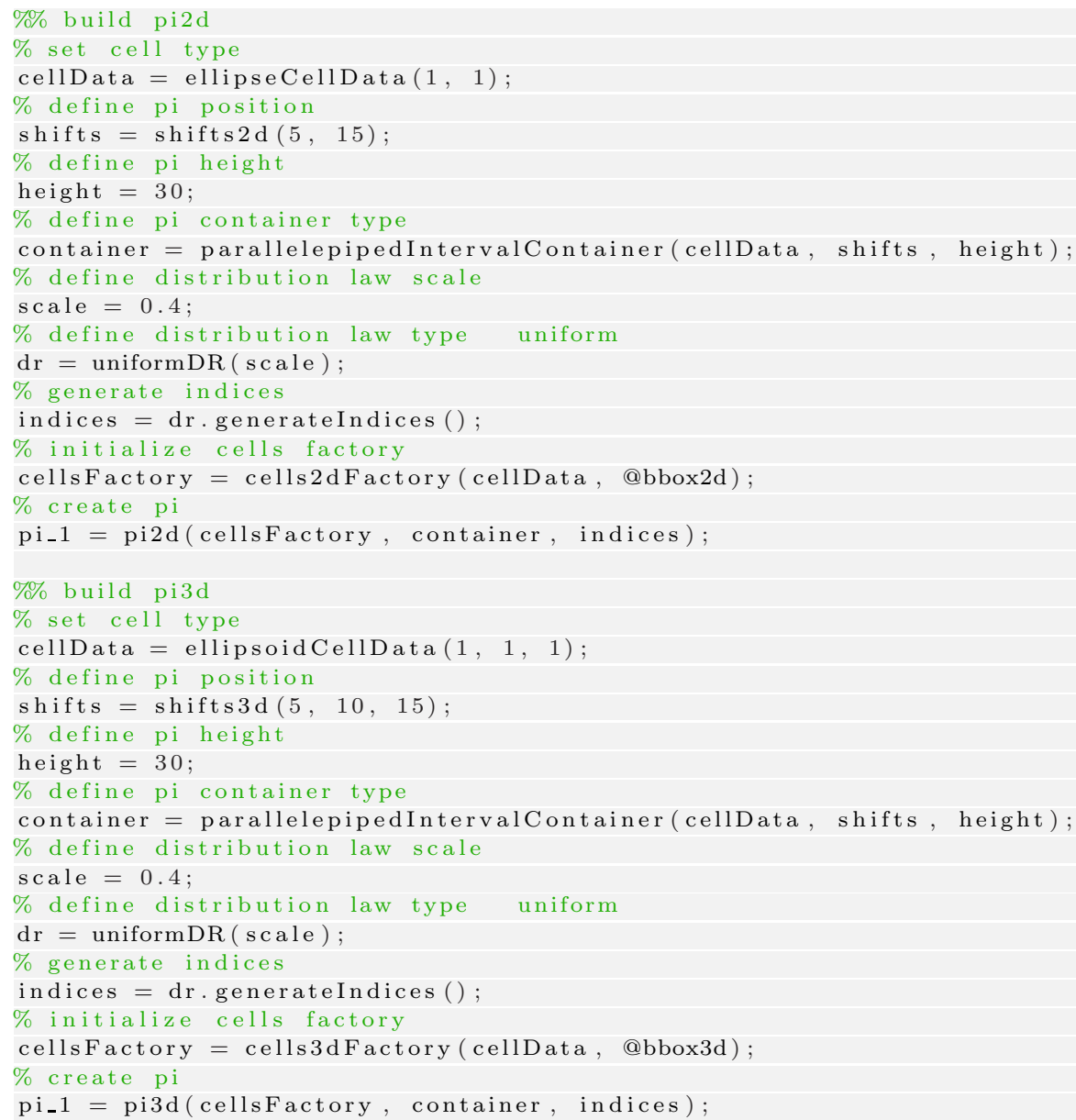



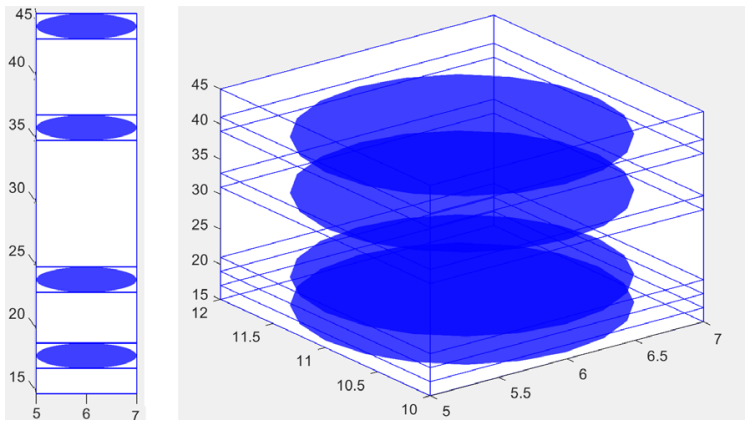

FIG. 3.1: Examples of $I_{p}$

\section{Distribution of Grains and Pores}

The distribution rule/law $\mu(x ; h, \delta y)$ plays a significant role for porous intervals and porous functions, as it is used to assign a cell (grain, pore) to a particular place. The PFT toolbox provides the drInterface class, which allows to use any mathematical algorithm for generating cell indices (random using any CDF - cumulative distribution function, or any deterministic distribution rule). Several classical CDFs (uniform distribution, normal distribution, etc.) are already implemented and can be used immediately.

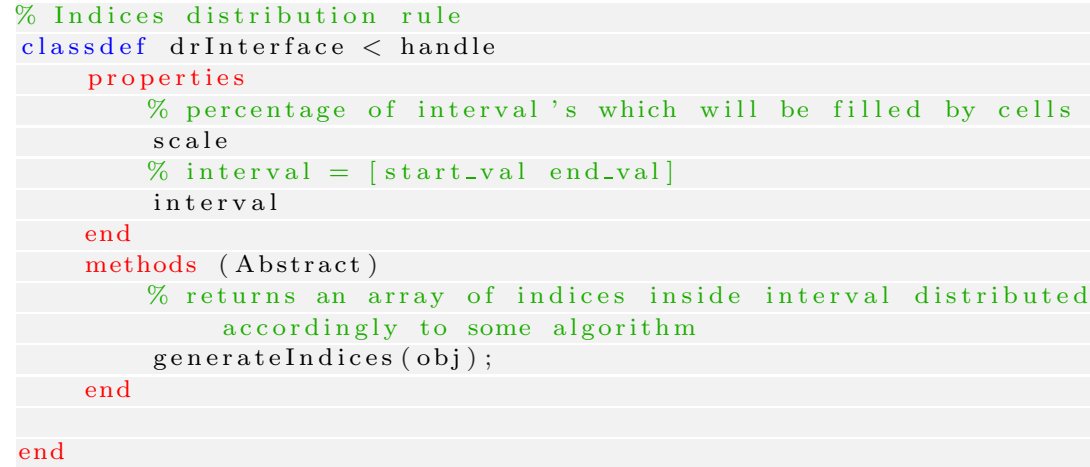

The distribution rule $\mu(x ; h, \delta y)$ uses the scale value $\rho \in[0,1]$ (the density of the distribution rule), that defines the percentage of $I_{p}$ filled by cells. The value of $\rho=1$ means that the porous interval is completely filled by cells (grains/pores). 


\section{Porous Functions}

5.1. Notation. A porous function $F_{p}$ is described as

$$
F_{p}=\left\{I_{p},\left[\begin{array}{l}
v(x) \\
u(x)
\end{array}\right], \phi, \mu(x ; h, \delta y)\right\}
$$

where $\phi$ is a function describing the cell type (a grain/pore shape and size).

In addition to the lower shape $u(x)$, , let us denote by $\eta$ the constraints of a physical container. The function $u(x)$ can be considered as one of the constraints, that $u(x) \in \eta$.

Using the setx notation, $F_{p}$ can be denoted as an indexed family of porous intervals $I_{p}$

$$
F_{p}=\left\{I_{p_{i}}\right\}_{i \in I}
$$

For a porous function of one variable we have:

$$
I=\left\{k: k \in D(v(x)) ; k_{m}=k_{m-1}+\delta x\right\},
$$

where $D(v(x))$ is the domain of a shape function $v(x)$.

For a porous function of two variables:

$$
I=\left\{(k, d): k, d \in D(v(x, y)) ; k_{m}=k_{m-1}+\delta x ; d_{n}=d_{n-1}+\delta y\right\},
$$

where $D(v(x, y))$ is the domain of a shape function $v(x, y)$.

In the case of a porous interval $I_{p}$, where every element of an indexed set $J$ links to a set of only one cell (3.4). However, the set of indices $I$ in (5.3) and (5.4) links to a set of porous intervals, which are located on the same spatial coordinates - the situation that occurs during shape or body addition of porous functions.

5.2. Creating porous functions in PFT. For creating or evaluating a porous function $F_{p}$ the following algorithm is used:

- define upper shape functions $v(x)$ [1] $(v(x, y)$ for $3 \mathrm{~d}$ case);

- define a distribution rule $\mu(x ; h, \delta y)(\mu(x, y ; h, \delta z)$ for $3 \mathrm{~d}$ case);

- define constraints $\eta$;

- define the cell shape and size $(\delta x, \delta y$ ( $\delta z$ for $3 \mathrm{~d}$ case) [1]);

- calculate the values of $v(x)(v(x, y)$ for $3 \mathrm{~d}$ case) for each value of $x$ ( $x, y$ for $3 \mathrm{~d}$ case) .

- for each value of the shape function build the corresponding porous interval $I_{p}$ as described in Section 3.2 .

In the PFT toolbox, this algorithm is implemented as a set of custom classes for handling porous intervals described earlier, and the $p f 2 d$ class ( $p f 3 d$ in the three-dimensional case), that includes the main properties and methods to initialize, build, draw and handle operations with porous functions: 


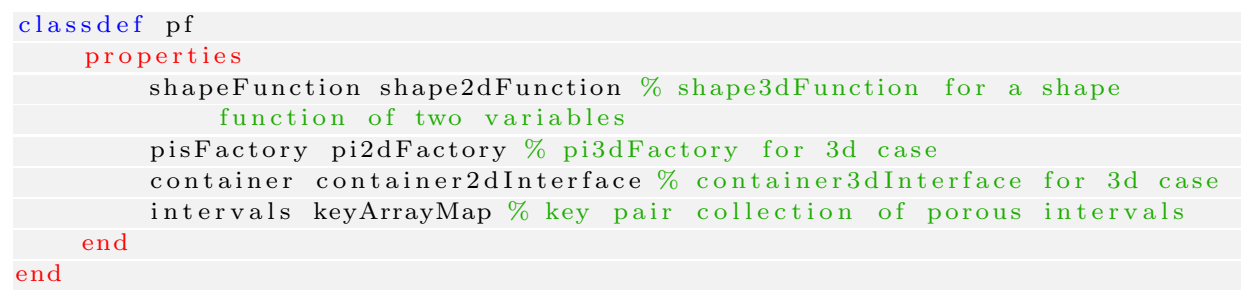

The shapeFunction property represents the shape function $v(x)$ of a porous function $F_{p}$. It can be of one variable or of two variables (implemented in shape2dFunction and shape3dFunction classes, respectively).

The purpose of the pisFactory property is similar to cellsFactory - it generates $I_{p}$ based on shapeFunction value for specific $x$ (or $x$ and $y$ in three-dimensional case), a distribution rule $\mu(x ; h, \delta y)$, and a cell type.

The container property plays the role of a 'physical container', that holds some porous material, described by $F_{p}$.

For creating a custom container, a new container class derived from container2dInterface (or, container3dInterface in the three-dimensional case) must be defined. In this way, any necessary type of an external $F_{p}$ container can be created.

The intervals property represents a custom data collection of $I_{p}$ of a type keyArrayMap, that is based on the built-in MATLAB type containers.Map, but with a possibility to use a numbered pair as a key to index every $I_{p}$ by its location coordinates ( $x$ in the two-dimensional case, $[x, y]$ in the three-dimensional case).

The porous function, generated by the following code snippet, is shown in Fig. 5.1:

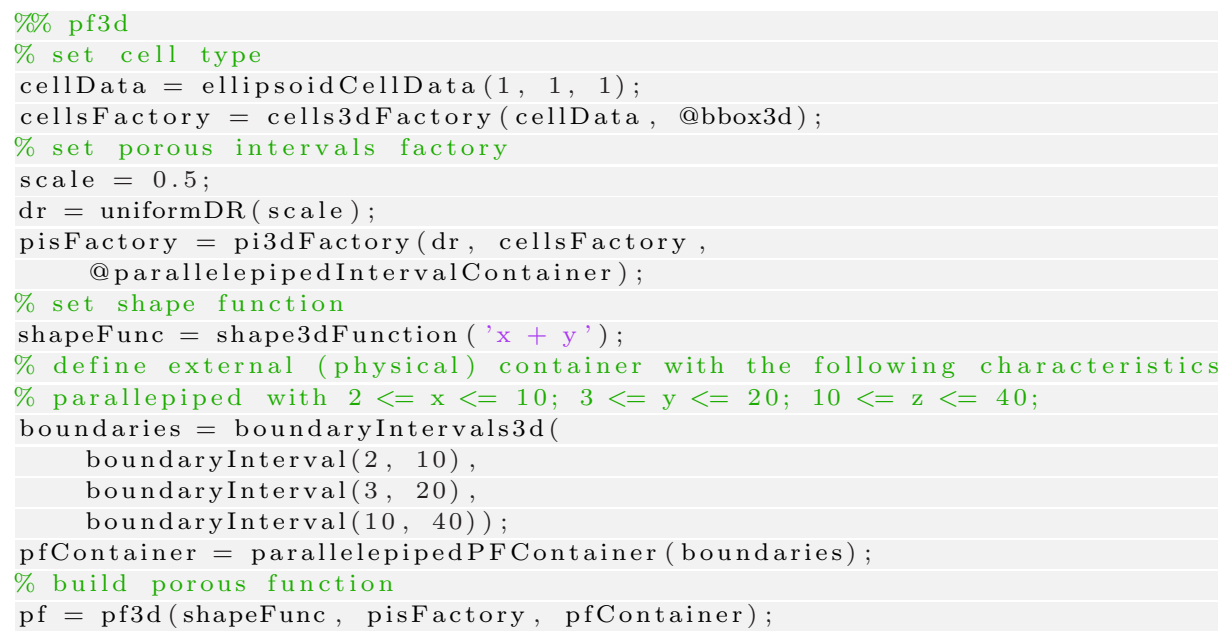




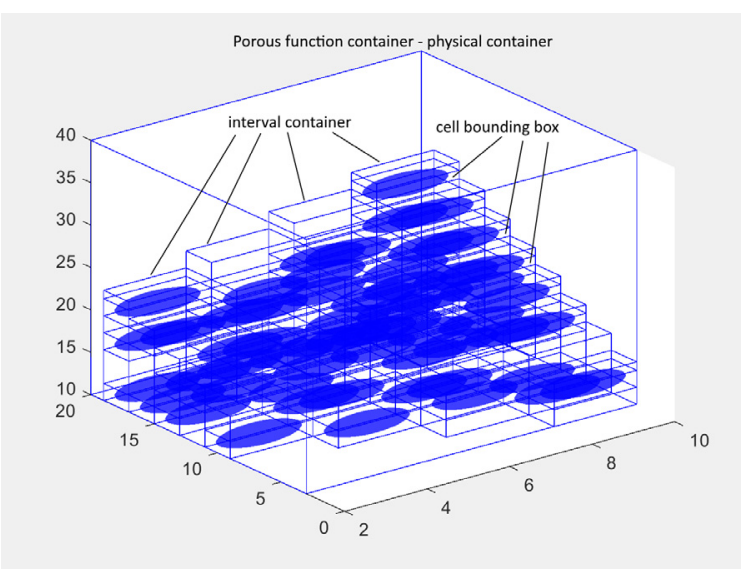

FIG. 5.1: Examples of $F_{p}$

As one can see in Fig. 5.1, a physical container can extend the maximum value of the shape function $\left(\max \left(v\left(x_{0}\right)\right)<\eta\left(x_{0}\right)\right)$, which means that there will be left some free space (not filled by cells). It can be filled later by another type of porous material (like in body addition). In the case when a physical container is smaller than the value of the shape function $\left(v\left(x_{0}\right)>\right.$ $\left.\eta\left(x_{0}\right)\right)$, the porous function is cropped to the level of the container border $\left(v\left(x_{0}\right)=\eta\left(x_{0}\right)\right)$.

It can occur that there will be some space not filled by grains or by pores; this happens due to the approximation of the shape function by a rectangular (or parallelepiped in three-dimensional case) porous intervals and the fact that a porous interval cannot always be completely filled by cells because the height of the porous interval can be divided by the height of a cell with a remainder.

\section{Equality of porous functions}

Definition 6.1. Consider two porous functions $F_{p}^{1}$ and $F_{p}^{2}$ :

$$
\begin{aligned}
& F_{p}^{1}=\left\{I_{p}^{1},\left[\begin{array}{l}
v^{1}(x) \\
u^{1}(x)
\end{array}\right], \psi^{1}, \mu^{1}\left(x ; h^{1}, \delta^{1} y\right)\right\}, \\
& F_{p}^{2}=\left\{I_{p}^{2},\left[\begin{array}{l}
v^{2}(x) \\
u^{2}(x)
\end{array}\right], \psi^{2}, \mu^{2}\left(x ; h^{2}, \delta^{2} y\right)\right\} .
\end{aligned}
$$

Two porous functions $F_{p}^{1}$ and $F_{p}^{2}$ are equal $\left(F_{p}^{1}=F_{p}^{2}\right)$, if all their characteristics are equal:

$$
I_{p}^{1}=I_{p}^{2},\left[\begin{array}{l}
v^{1}(x) \\
u^{1}(x)
\end{array}\right]=\left[\begin{array}{l}
v^{2}(x) \\
u^{2}(x)
\end{array}\right], \psi^{1}=\psi^{2}, \mu^{1}\left(x ; h^{1}, \delta^{1} y\right)=\mu^{2}\left(x ; h^{2}, \delta^{2} y\right) .
$$



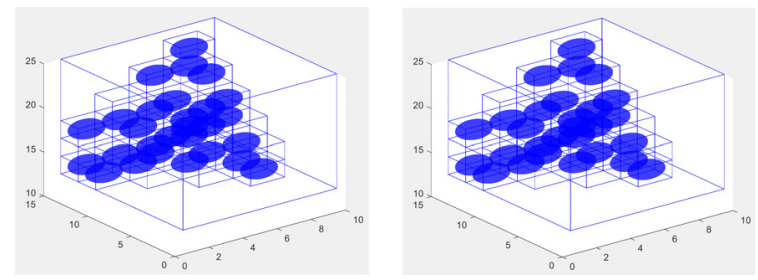

FiG. 6.2: Absolutely equal porous functions

As a porous function can include randomization due to the use of a distribution law/rule, two porous functions can be equal in the sense of Def. 6.1, but their visual representations can differ. Thus, let us define the absolute equality, when in addition to the previous the equality of all cell locations is required.

Definition 6.2. Two porous functions $F_{p}^{1}$ and $F_{p}^{2}$ are absolutely equal $\left(F_{p}^{1} \equiv F_{p}^{2}\right)$, if

$$
F_{p}^{1}=F_{p}^{2}, \quad I^{1}=I^{2},
$$

where $I^{i}(\underline{(5.2)})$ are compared in the terms of the equality of sets.

In Fig. 6.1 two equal porous functions are depicted. It is easily seen, cels have different values: grains and pores occupy different cells of the same porous intervals. In Fig. 6.2 two absolutely equal porous functions are shown: all grains and pores are at the same positions.
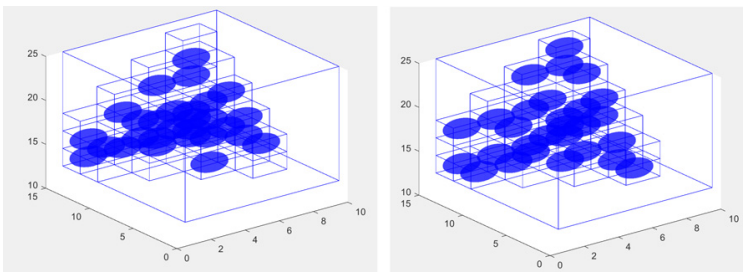

FIG. 6.1: Equal porous functions

Every time the build function of $p f$ class is called after the creation of porous interval using some probabilistic distribution law, new cell indices are generated, hence equal (but not absolutely equal) porous functions are built. 


\section{Addition of Porous Functions}

Two possible approaches to addition of porous functions were outlined in [1]: the shape addition and the body addition.

7.1. Shape addition. Consider two porous functions $F_{p}^{1}$ and $F_{p}^{2}$ :

$$
\begin{aligned}
& F_{p}^{1}=\left\{I_{p_{i}}^{1}\right\}_{i \in I^{1}}, \\
& F_{p}^{2}=\left\{I_{p_{i}}^{2}\right\}_{i \in I^{2}},
\end{aligned}
$$

Definition 7.1. Shape addition $\cup^{s}$ is defined as

where

$$
F_{p}^{1} \cup^{s} F_{p}^{2}=\left\{I_{p_{i}}^{1} \cup^{s} I_{p_{i}}^{2}\right\}_{i \in I^{1} \cup^{s} I^{2}},
$$

$$
\begin{gathered}
I_{p_{i}}^{1} \cup^{s} I_{p_{i}}^{2}=\left\{C_{j}\right\}_{j \in J^{1}} \cup^{s}\left\{C_{j}\right\}_{j \in J^{2}}=\left\{C_{j}\right\}_{j \in J^{1} \cup J^{2}+h}, \\
I^{1} \cup^{s} I^{2}=I^{1} \cup I^{2} \cap I_{\eta},
\end{gathered}
$$

and $I_{\eta}$ is the set of all possible indices of porous intervals in the $\eta$ container.

The shape addition of porous functions is based on the shape addition of its porous intervals in the corresponding positions. One porous function can be defined on the wider, bigger container, meaning that some (or even all) interval positions can differ.

If the first porous function is "wider", it does not create any problem, because all porous intervals of the second porous function can be added. However, if the height of these intervals exceeds the external container, it will be cropped to the container boundaries. In the second porous function is "wider", its intervals are cropped to the container boundaries in all directions.

The following code snippet illustrates how the shape addition of two porous functions $F_{p}^{1}$ and $F_{p}^{2}$ is performed with the help of the method addShape provided by the class $p f$ of the PFT toolbox. The result is shown in Fig. 7.1.

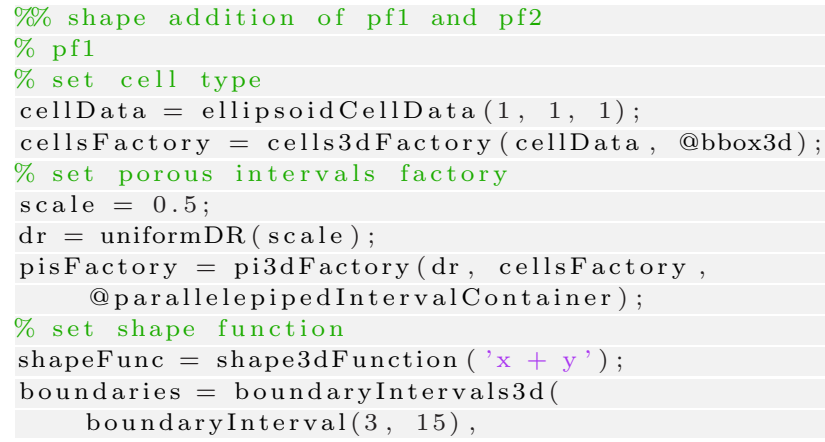



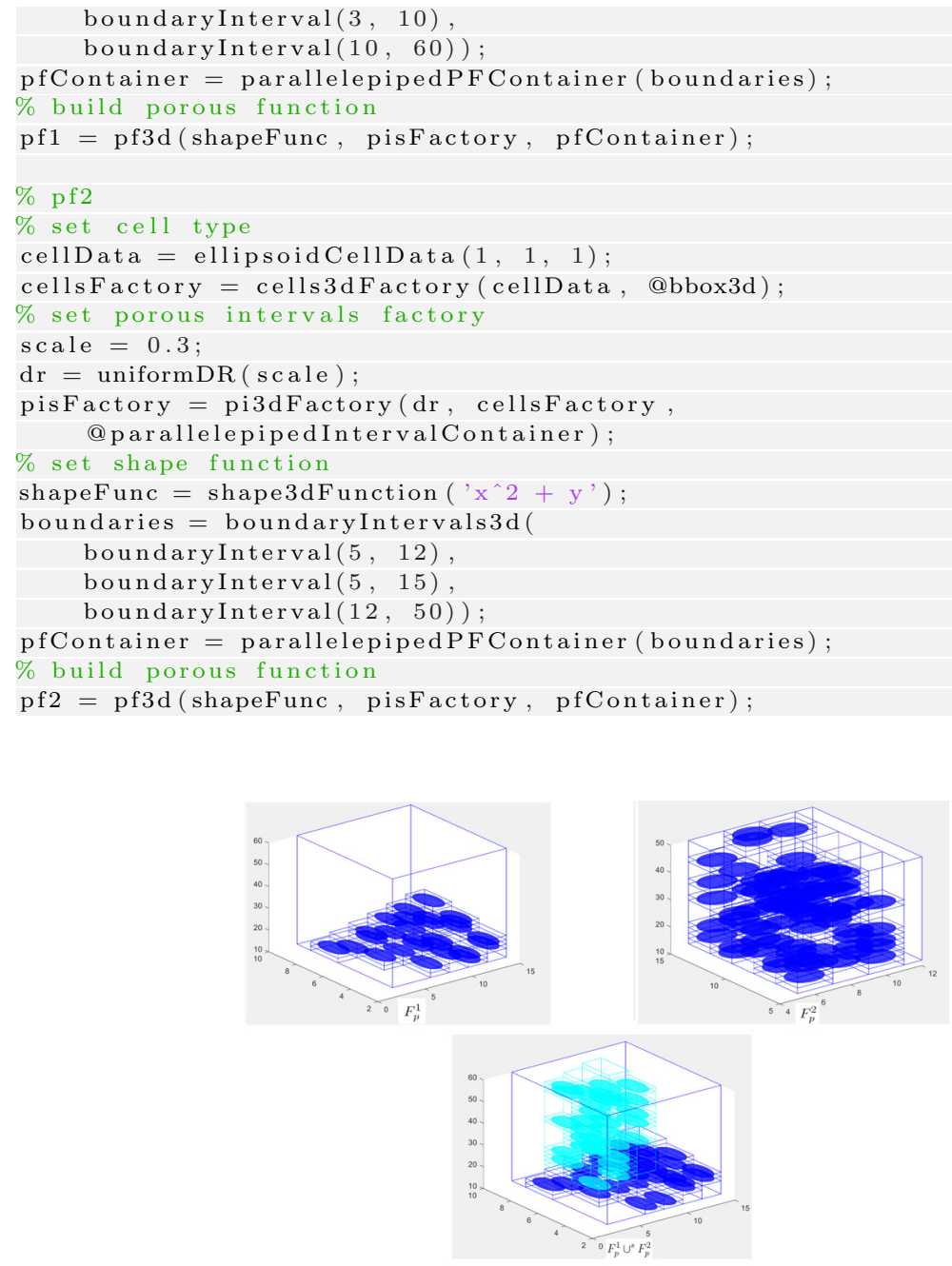

FIG. 7.1: Shape addition of $F_{p}^{1}$ and $F_{p}^{2}$

It is important to note that the shape addition is a commutative operation only in terms of equality (Def. 6.1) only if both porous functions have the same distribution rule and cell type.

Although in general the shape addition is not commutative, it describes the natural situation of adding two different porous (or granular) materials.

7.2. Body addition. Another type of addition [1]) is the body addition. It is somewhat similar to a point-wise addition of matrices. The main idea 
is to fill the empty space of the first porous function by the cells of the second porous function.

Consider $F_{p}^{1}$ and $F_{p}^{2}$ :

$$
\begin{aligned}
& F_{p}^{1}=\left\{I_{p_{i}}^{1}\right\}_{i \in I^{1}}, \\
& F_{p}^{2}=\left\{I_{p_{i}}^{2}\right\}_{i \in I^{2}},
\end{aligned}
$$

Definition 7.2. Body addition $\cup^{b}$ of $F_{p}^{1}$ and $F_{p}^{2}$ is defined as

$$
\begin{gathered}
F_{p}^{1} \cup^{b} F_{p}^{2}=\left\{I_{p_{i}}^{1} \cup^{b} I_{p_{i}}^{2}\right\}_{i \in I^{1} \cup b I^{2}}, \\
I^{1} \cup^{b} I^{2}=I^{1}, \\
I_{p_{i}}^{1} \cup^{b} I_{p_{i}}^{2}=\left\{C_{j}\right\}_{j \in J^{1}} \cup^{b}\left\{C_{j}\right\}_{j \in J^{2}}=\left\{C_{j}\right\}_{j \in J^{1} \cup\left(J^{2} \backslash J^{1}\right) \cup \Phi\left(J^{1} \cap J^{2}\right)},
\end{gathered}
$$

where $\Phi(J)$ is a relation producing the closest unused index (used for the cells of the second addend, whose corresponding locations in the first addend are taken).

In Fig. 7.2 the result of the body addition of two porous functions is shown for the same functions as in Section 7.1.

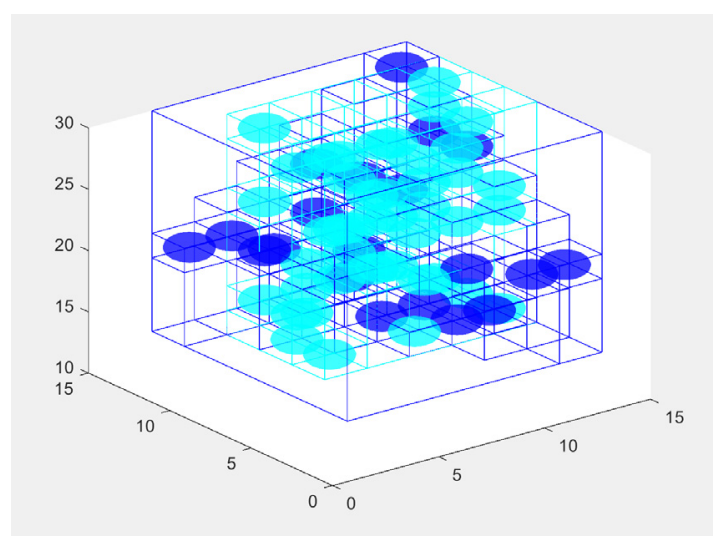

FIG. 7.2: Body addition

\section{Subtraction of Porous Functions}

The main idea of subtracting one porous function from another consists of removing some cells of one porous function from the other. This operation is similar to the set difference $(\backslash)$. 
Definition 8.1. For $F_{p}^{1}$ defined by (7.6) and $F_{p}^{2}$ defined by (7.7)

$$
F_{p}^{1}-F_{p}^{2}=F_{p}^{1} \backslash F_{p}^{2}=\left\{I_{p_{i}}^{1} \backslash I_{p_{i}}^{2}\right\}_{i \in I^{1} \backslash I^{2}} .
$$

The following code snippet illustrates the operation of subtraction of two porous functions, and the result is shown in Fig. 8.1.

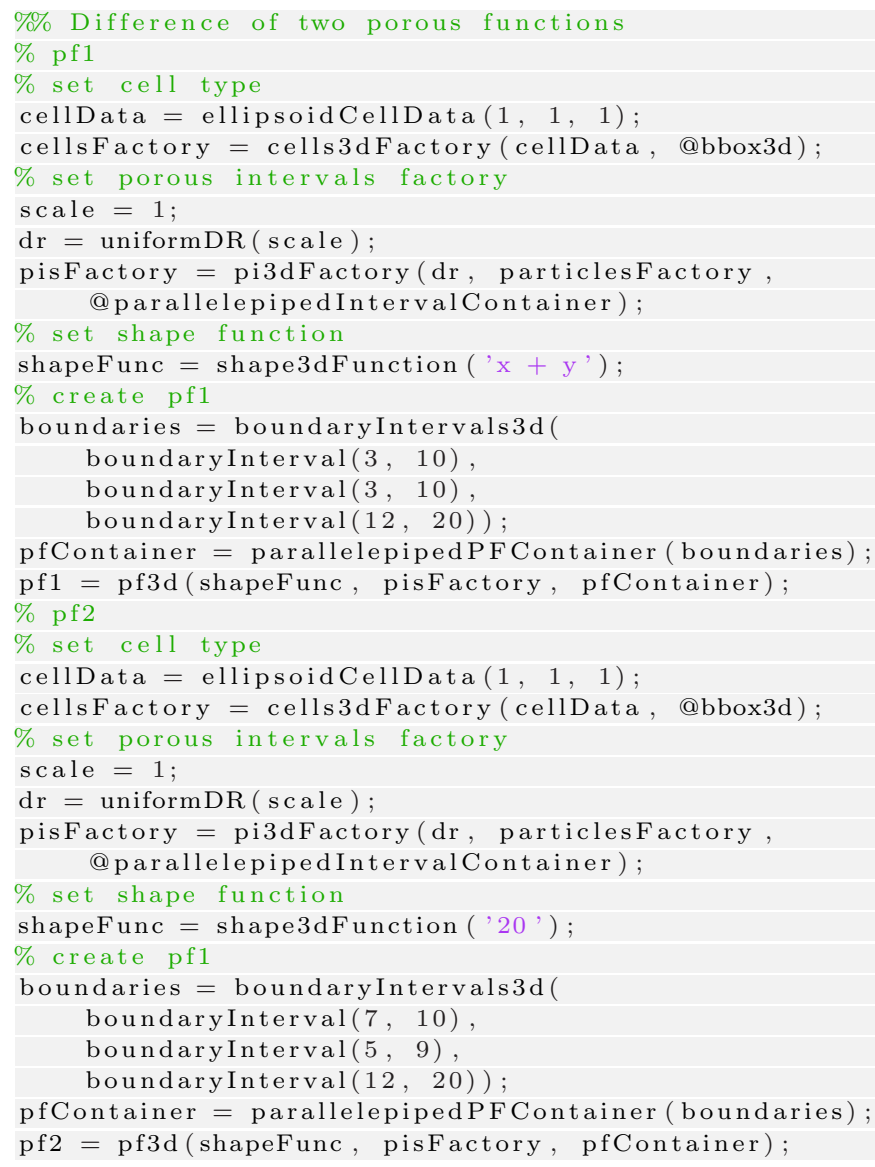

Such definition of subtraction satisfies $F_{p}^{1}-F_{p}^{1}=F_{p}^{0}$, where $F_{p}^{0}$ is a zero porous function - the height of all its porous intervals is zero.

\section{Multiplication by a Scalar}

The multiplication of a porous function by a scalar modifies its shape by setting the height of each its porous interval by its replication. For $n \in N$ we have simply

$$
\begin{gathered}
n * F_{p}=\left\{n * I_{p_{i}}\right\}_{i \in I}, \\
n * I_{p}=\left\{C_{j}\right\}_{j \in J \cup_{p=1}^{n}(J+p * h)} .
\end{gathered}
$$



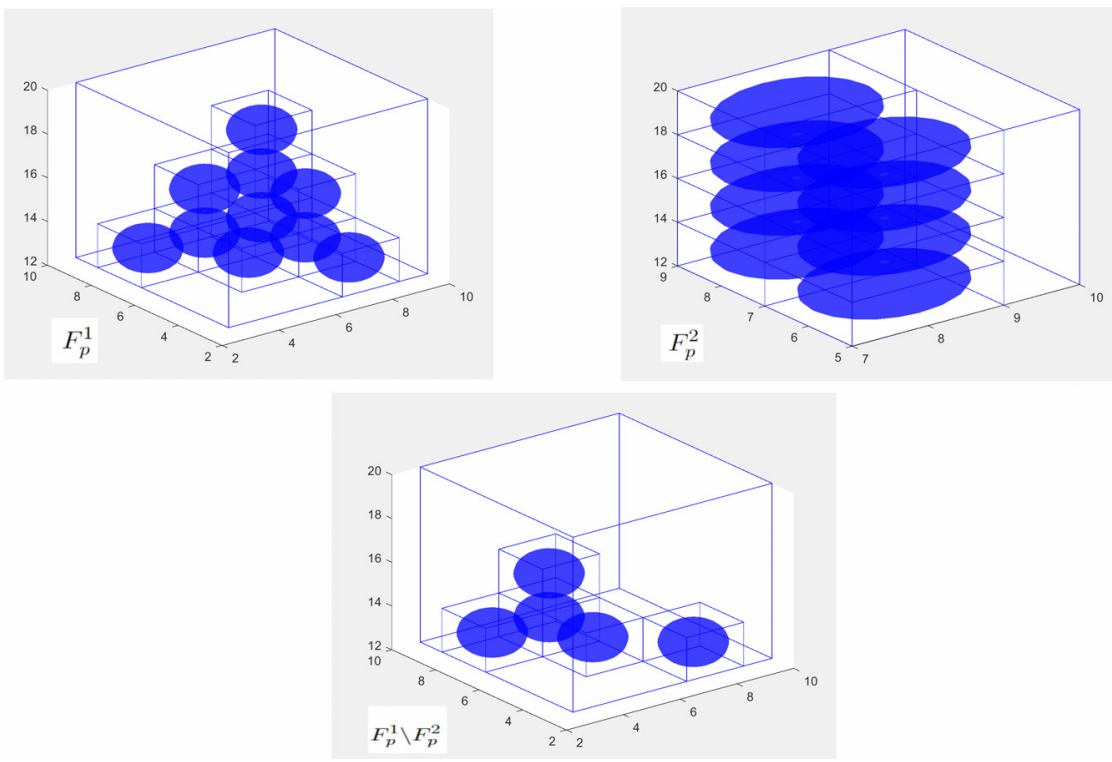

FIG. 8.1: Subtraction of porous functions

An example of scalar multiplication of $F_{p}^{1}$ defined in Section 8 by $n=2$ can be seen on Fig. 9.1.

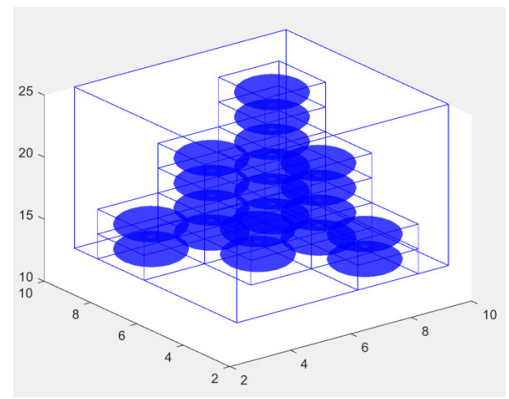

FIG. 9.1: Multiplication by a scalar: $n F_{p}^{1}$ for $n=2$

\section{Conclusion}

In this paper, we have presented basic operations with porous functions in more detail, and provided necessary computational tools in the form of the Porous Functions Toolbox for MATLAB. 
The current implementation of this toolbox allows the creation of porous functions of one and two variables, with various constraints, with various distribution laws/rules (uniform, normal distribution, etc.) defining positions of grains/pores of a porous function, with cells of various shapes and sizes for placement of grains and pores.

The Porous Functions Toolbox is based on the object-oriented programming paradigms, allowing for easy extension and customization of the existing functionality.

The main purpose of the Porous Functions Toolbox is to provide an easily understandable and flexible tool for modeling the structure of various real-world materials (porous, granular, composite, ...) and for performing basic operations with porous functions for their applications in science and engineering.

\section{Acknowledgements}

This work was supported in parts by grants APVV-18-0526, APVV-140892, APVV SK-SRB-18-0011, VEGA 1/0365/19, COST CA15225.

\section{References}

[1] I. Podlubny, Porous functions. Fract. Calc. Appl. Anal. 22, No 6 (2019), 1502-1516; DOI: 10.1515/fca-2019-0078; https://www.degruyter.com/view/journals/fca/22/6

/fca.22.issue-6.xml.

[2] I. Trymorush, A new class of functions for applications in modeling real materials and structures. In: ICCM'2018 - The 9th International Conference on Computational Methods, August 6-10, 2018, Rome, Italy.

[3] I. Podlubny, Modeling of real materials: time and structure. In: ICFCTA'2019 - International Conference of the Fractional Calculus Theory and Applications, April 25-26, 2019, Bourges, France.

[4] I. Podlubny, Porous functions: a new tool for modeling real materials. In: 4-th International Conference on Applied Fractional Calculus, July 8, 2019, Beijing, China.

[5] I. Trymorush, Modeling and control of transfer processes in real materials. Internal Report, FBERG, Technical University of Kosice, December $20,2018$.

[6] I. Trymorush, Porous functions toolbox. MATLAB Central File Exchange, Submission 74874 (Retrieved April 5, 2020); https://www.mathworks.com/matlabcentral/fileexchange/74874. 
BERG Faculty, Technical University of Kosice

B. Nemcovej 3

04200 Kosice, SLOVAKIA

e-mail: iryna.trymorush@tuke.sk;

igor.podlubny@tuke.sk

Received: December 20, 2019

Please cite to this paper as published in:

Fract. Calc. Appl. Anal., Vol. 23, No 1 (2020), pp. 307-323,

DOI: 10.1515/fca-2020-0015; at https://www.degruyter.com/view/j/fca. 